\section{Management-Seminar der SGC für Ärzte mit Führungsverantwortung der Union Chirurgischer Fachgesellschaften}

Unter dem Patronat der Schweizerischen Gesellschaft für Chirurgie (SGC) wird eine weitere Auflage des bisher sehr erfolgreichen Managementkurses organisiert, der zum ersten Mal nicht nur Mitgliedern der SGC, sondern neu auch Chefärztinnen und -ärzten sowie leitenden Ärztinnen und Ärzten aller Disziplinen der Union Chirurgischer Fachgesellschaften (Union) offensteht.

\section{Themen und Inhalte sind in 3 Kreisen lokalisiert}

Führung und Organisation

Strategieentwicklung, Change-Management, Führungsorganisation, Führungsstile und -rollen, Human-Resource-Management, Informationsfluss gestalten; Spitalkultur entwickeln, Spital nach aussen vertreten.

\section{Führung anderer}

Wirkungsvoll kommunizieren; resultatorientiert arbeiten; Personal auswählen, bewerten, entwickeln und leiten; Teams aufbauen und Leistung fördern, Konflikte konstruktiv gestalten; schwierige Arbeitsbeziehungen positiv beeinflussen.

\section{Selbstführung}

Gesunden Lebensstil entwickeln, Umgang mit Stress und Fehlleistungen, Zeitmanagement, eigene Ressourcen mehren und gezielt einsetzen, Persönlichkeits- und Laufbahnentwicklung.

S.

Anmeldung zum Kurs

Name:

Vorname:

Adresse:

PLZ/Ort:

Telefon/Fax:

\section{Teilnehmende}

Das Angebot richtet sich gezielt an Chefärztinnen und Chefärzte sowie leitende Ärztinnen und Ärzte mit Führungserfahrung. Aus didaktischen Gründen bleibt die Teilnehmerzahl auf 16 beschränkt, damit die persönlichen Ansprüche der einzelnen Kursteilnehmer/ Innen zum Zug kommen können.

Das Managementseminar ist eine anerkannte Fortbildung der SGC und wird mit 50 SGC-RatingPoints bewertet.

\section{Kursstruktur}

Vorgesehen sind drei Weiterbildungsblöcke (einmal 3 und zweimal 2 Tage). Es wird an praktischen (eigenen) kritischen Erfahrungen und Fragestellungen angeknüpft und Theorie anhand konkreter Fallbeispiele entwickelt. Dialog und Kleingruppenarbeit stehen im Zentrum eines Lernprozesses, der von den Teilnehmenden und der Leitung gemeinsam gestaltet wird.

\section{Seminardaten}

27.-29. November 2000

22./23. Januar 2001

23./24. April 2001

\section{Kosten}

Der Managementkurs ist als Einheit konzipiert und kostet pro Teilnehmer Fr. 3500.- (inkl. Kursunterlagen), zuzüglich Kosten für Verpflegung und Unterkunft.

\section{Leitung}

Elisabeth Michel-Alder, Inhaberin der Unternehmungsberatungsfirma ema Human Potential Development, in Zürich. Sie ist Sozialwissenschaftlerin, Organisationsentwicklerin und (auch im Spitalbereich) erfahrene Managementtrainerin.

\section{Organisation}

Schweizerische Gesellschaft für Chirurgie Die schriftliche Anmeldung mit einem kurzen Lebenslauf sind einzusenden an: Dr. med. Jean Biaggi, Egghölzliweg 4 a, 3074 Muri, Tel. 03195172 34, Fax 0319517210.

Sekretariat Management-Seminar, Frau F. Fischer, Sägematt 5, 3507 Biglen, Tel. 0317012757. 